Also available at http://amc.imfm.si

ISSN 1855-3966 (printed edn.), ISSN 1855-3974 (electronic edn.)

ARS MATHEMATICA CONTEMPORANEA 6 (2013) 117-125

\title{
Some properties of the Zagreb eccentricity indices
}

\author{
Kinkar Ch. Das * \\ Department of Mathematics, Sungkyunkwan University \\ Suwon 440-746, Republic of Korea \\ Dae-Won Lee \\ Sungkyunkwan University, Suwon 440-746, Republic of Korea \\ Ante Graovac \\ Faculty of Science, University of Split, Nikole Tesle 12, HR-21000 Split, Croatia
}

Received 10 October 2011, accepted 20 January 2012, published online 5 June 2012

\section{Abstract}

The concept of Zagreb eccentricity $\left(E_{1}\right.$ and $\left.E_{2}\right)$ indices was introduced in the chemical graph theory very recently $[5,12]$. The first Zagreb eccentricity $\left(E_{1}\right)$ and the second Zagreb eccentricity $\left(E_{2}\right)$ indices of a graph $G$ are defined as

$$
E_{1}=E_{1}(G)=\sum_{v_{i} \in V(G)} e_{i}^{2}
$$

and

$$
E_{2}=E_{2}(G)=\sum_{v_{i} v_{j} \in E(G)} e_{i} \cdot e_{j},
$$

where $E(G)$ is the edge set and $e_{i}$ is the eccentricity of the vertex $v_{i}$ in $G$. In this paper we give some lower and upper bounds on the first Zagreb eccentricity and the second Zagreb eccentricity indices of trees and graphs, and also characterize the extremal graphs.

Keywords: Graph, first Zagreb eccentricity index, second Zagreb eccentricity index, diameter, eccentricity.

Math. Subj. Class.: 05C40, 05C90

\footnotetext{
* Corresponding author

E-mail addresses: kinkardas2003@googlemail.com (Kinkar Ch. Das), haverd2001@gmail.com (Dae-Won Lee), ante.graovac@irb.hr (Ante Graovac)
} 


\section{Introduction}

Mathematical chemistry is a branch of theoretical chemistry using mathematical methods to discuss and predict molecular properties without necessarily referring to quantum mechanics $[1,8,14]$. Chemical graph theory is a branch of mathematical chemistry which applies graph theory in mathematical modeling of chemical phenomena [6]. This theory has an important effect on the development of the chemical sciences.

Topological indices are numbers associated with chemical structures derived from their hydrogen-depleted graphs as a tool for compact and effective description of structural formulas which are used to study and predict the structure-property correlations of organic compounds. Molecular descriptors are playing significant role in chemistry, pharmacology, etc. Among them, topological indices have a prominent place [13]. One of the best known and widely used is the connectivity index, $\chi$, introduced in 1975 by Milan Randić [11]. The Randić index is one of the most famous molecular descriptors and the paper in which it is defined is cited more than 1000 times. The first $M_{1}$, and the second $M_{2}$, Zagreb indices (see [2],[3],[4],[7],[9] and the references therein) are defined as:

$$
M_{1}=M_{1}(G)=\sum_{v_{i} \in V(G)} d_{i}^{2}
$$

and

$$
M_{2}=M_{2}(G)=\sum_{v_{i} v_{j} \in E(G)} d_{i} \cdot d_{j} .
$$

where $d_{i}$ is the degree of the vertex $v_{i} \in V(G)$ in $G$.

Let $G=(V, E)$ be a connected simple graph with $|V(G)|=n$ vertices and $|E(G)|=$ $m$ edges. Also let $d_{i}$ be the degree of the vertex $v_{i}, i=1,2, \ldots, n$. For vertices $v_{i}, v_{j} \in$ $V(G)$, the distance $d_{G}\left(v_{i}, v_{j}\right)$ is defined as the length of the shortest path between $v_{i}$ and $v_{j}$ in $G$. The eccentricity of a vertex is the maximum distance from it to any other vertex,

$$
e_{i}=\max _{v_{j} \in V(G)} d_{G}\left(v_{i}, v_{j}\right) .
$$

The maximum eccentricity over all vertices of $G$ is called the diameter of $G$ and denoted by $d$.

The invariants based on vertex eccentricities attracted some attention in Chmistry. In an analogy with the first and the second Zagreb indices, M. Ghorbani et al. and D. Vukičević et al. define the first $E_{1}$, and the second, $E_{2}$, Zagreb eccentricity indices by [5, 12]

$$
E_{1}=E_{1}(G)=\sum_{v_{i} \in V(G)} e_{i}^{2}
$$

and

$$
E_{2}=E_{2}(G)=\sum_{v_{i} v_{j} \in E(G)} e_{i} \cdot e_{j} .
$$

where $E(G)$ is the edge set and $e_{i}$ is the eccentricity of the vertex $v_{i}$ in $G$.

Let $G=(V(G), E(G))$. If $V(G)$ is the disjoint union of two nonempty sets $V_{1}(G)$ and $V_{2}(G)$ such that every vertex in $V_{1}(G)$ has degree $r$ and every vertex in $V_{2}(G)$ has degree 
$s$, then $G$ is $(r, s)$-semiregular graph. When $r=s$, is called a regular graph. As usual, $K_{a, b}(a+b=n), P_{n}$ and $K_{1, n-1}$ denote respectively the complete bipartite graph, the path and the star on $n$ vertices. A vertex of a graph is said to be pendent if its neighborhood contains exactly one vertex. An edge of a graph is said to be pendent if one of its vertices is a pendent vertex. Now we calculate

$$
E_{1}\left(P_{n}\right)= \begin{cases}\frac{1}{12}(n-1)\left(7 n^{2}-2 n\right) & \text { if } n \text { is even } \\ \frac{1}{12}(n-1)\left(7 n^{2}-2 n-3\right) & \text { if } n \text { is odd }\end{cases}
$$

and

$$
E_{2}\left(P_{n}\right)= \begin{cases}\frac{1}{12} n\left(7 n^{2}-21 n+20\right) & \text { if } n \text { is even } \\ \frac{1}{12}(n-1)\left(7 n^{2}-14 n+3\right) & \text { if } n \text { is odd. }\end{cases}
$$

Also we have

$$
E_{1}\left(K_{1, n-1}\right)=4 n-3 \text { and } E_{2}\left(K_{1, n-1}\right)=2 n-2 .
$$

Denote by $\tilde{T}_{n}$, is a tree of order $n$ with maximum degree $n-2$. We have $E_{1}\left(\tilde{T}_{n}\right)=$ $9 n-10, E_{2}\left(\tilde{T}_{n}\right)=6 n-8$.

In this paper we give some lower and upper bounds on the first Zagreb eccentricity and the second Zagreb eccentricity indices of trees and graphs, and also characterize the extremal graphs.

\section{Lower and upper bounds on Zagreb eccentricity indices}

We now give lower and upper bounds on the Zagreb eccentricity indices of trees.

Theorem 2.1. Let $T$ be a tree with $n$ vertices. Then

$$
\text { and (ii) } \quad E_{2}\left(K_{1, n-1}\right) \leq E_{2}(T) \leq E_{2}\left(P_{n}\right) \text {. }
$$

Moreover, the left hand side (right hand side, respectively) equality holds in (2.1) and (2.2) if and only if $G \cong K_{1, n-1}\left(G \cong P_{n}\right.$, respectively).

Proof. Upper bound: If $T$ is isomorphic to path $P_{n}$, then the right hand side equality holds in (2.1) and (2.2). Otherwise, $T \supsetneqq P_{n}$. Let $d$ be the diameter of tree $T$. Then there exists a path $P_{d+1}: v_{1} v_{2} \ldots v_{d+1}$ of length $d$ in $T$. Thus we have the eccentricity of a vertex $v_{i}$ in tree $T$,

$$
e_{i}=\max \left\{d_{G}\left(v_{i}, v_{1}\right), d_{G}\left(v_{i}, v_{d+1}\right)\right\} .
$$

Since $T$ is a tree, both vertices $v_{1}$ and $v_{d+1}$ are pendent vertices. Thus we have $e_{i} \leq d$ for each $v_{i} \in V(G)$. Since $T ¥ P_{n}$, let $v_{k}(k \neq 1, d+1)$ be a vertex of degree one, adjacent to vertex $v_{j}$ in $T$. We transform $T$ into another tree $T^{*}$ by deleting the edge $v_{k} v_{j}$ and join the vertices $v_{d+1}$ and $v_{k}$ by an edge. Then the longest path $P_{d+2}: v_{1} v_{2} \ldots v_{d+1} v_{k}$ of length $d+1$ in $T^{*}$. Let the vertex eccentricities be $e_{1}^{*}, e_{2}^{*}, \ldots, e_{n}^{*}$ in $T^{*}$. Therefore we have $e_{t}^{*}=\max \left\{d_{G}^{*}\left(v_{t}, v_{1}\right), d_{G}^{*}\left(v_{t}, v_{k}\right)\right\}=\max \left\{d_{G}\left(v_{t}, v_{1}\right), d_{G}\left(v_{t}, v_{d+1}\right)+1\right\} \geq$ $\max \left\{d_{G}\left(v_{t}, v_{1}\right), d_{G}\left(v_{t}, v_{d+1}\right)\right\}=e_{t}\left(\right.$ as $\left.d_{G}^{*}\left(v_{t}, v_{k}\right)=d_{G}\left(v_{t}, v_{d+1}\right)+1\right)$ for $t \neq k$ whereas $e_{k}^{*}=d+1>d \geq e_{k}\left(d_{G}^{*}\left(v_{i}, v_{j}\right)\right.$ is the length of the shortest path between vertices 
$v_{i}$ and $v_{j}$ in $T^{*}$ ). So we have $e_{r}^{*} e_{s}^{*} \geq e_{r} e_{s}$ for $v_{r} v_{s} \neq v_{k} v_{j}, v_{k} v_{d+1}$ and $e_{k}^{*} e_{d+1}^{*}=$ $d(d+1)>d^{2} \geq e_{k} e_{j}$. Using above result we get

$$
E_{1}\left(T^{*}\right)-E_{1}(T)=\sum_{v_{i} \in V\left(T^{*}\right)} e_{i}^{* 2}-\sum_{v_{i} \in V(T)} e_{i}^{2} \geq e_{k}^{* 2}-e_{k}^{2}>0
$$

and

$$
E_{2}\left(T^{*}\right)-E_{2}(T)=\sum_{v_{r} v_{s} \in E\left(T^{*}\right)} e_{r}^{*} e_{s}^{*}-\sum_{v_{r} v_{s} \in E(T)} e_{r} e_{s} \geq e_{k}^{*} e_{d+1}^{*}-e_{k} e_{j}>0 .
$$

Therefore we have

$$
E_{i}\left(T^{*}\right)>E_{i}(T), \quad i=1,2 .
$$

By the above described construction we have increased the value of $E_{i}(T), i=1,2$. If $T^{*}$ is the path, we are done. If not, then we continue the construction as follows. Next we choose one pendent vertex $\left(\neq v_{1}, v_{k}\right)$ from $T^{*}$, etc. Repeating the procedure sufficient number of times, we arrive at a tree in which the maximum degree 2 , that is, we arrive at path $P_{n}$.

Lower bound: If $T$ is isomorphic to star $K_{1, n-1}$, then the left hand side equality holds in (2.1) and (2.2). If $T$ is isomorphic to $\tilde{T}_{n}$, then the left hand side inequality is strict in (2.1) and (2.2). Otherwise, $T ¥ K_{1, n-1}, \tilde{T}_{n}$. Suppose that a path $P_{d+1}: v_{1} v_{2} \ldots v_{d+1}$ of length $d$ in $T$, where $d$ is the diameter of $T$. Without loss of generality, we can assume that $d_{2} \geq d_{d}$ (the degree of vertex $v_{2}$ is greater than or equal to the degree of vertex $v_{d}$ ). Now choose $v_{i}$ to be an arbitrary maximum degree vertex, unless $v_{d}$ has maximum degree, in which case $v_{i}$ is chosen to be $v_{2}$. We transform $T$ into another tree $\hat{T}$ by deleting the edge $v_{d} v_{d+1}$ and join the vertices $v_{i}$ and $v_{d+1}$ by an edge. Let the vertex eccentricities be $\hat{e}_{1}, \hat{e}_{2}, \ldots, \hat{e}_{n}$ in tree $\hat{T}$. Similarly, as before we obtain $\hat{e}_{t} \leq e_{t}$ for all $t=1,2, \ldots, n$. Using above we get

$$
E_{1}(\hat{T})-E_{1}(T)=\sum_{v_{i} \in V(\hat{T})} \hat{e}_{i}^{2}-\sum_{v_{i} \in V(T)} e_{i}^{2} \leq 0
$$

and

$$
E_{2}(\hat{T})-E_{2}(T)=\sum_{v_{r} v_{s} \in E(\hat{T})} \hat{e}_{r} \hat{e}_{s}-\sum_{v_{r} v_{s} \in E(T)} e_{r} e_{s} \leq 0
$$

Therefore we have

$$
E_{i}(\hat{T}) \leq E_{i}(T), \quad i=1,2
$$

By the above described construction we have non-increased the value of $E_{i}(T), i=$ 1,2 . If $\hat{T}$ is to the tree $\tilde{T}_{n}$, we are done. If not, then we continue the construction as follows. Next we choose one pendent vertex from longest path in $\hat{T}$ such that its adjacent vertex is not maximum degree vertex. Now we delete that pendent edge and join the pendent vertex to the maximum degree vertex, etc. Repeating the procedure sufficient number of times, we arrive at a tree in which the maximum degree $n-2$, that is, we arrive at tree $\tilde{T}_{n}$. This completes the proof. 
We now give lower and upper bounds on the Zagreb eccentricity indices of bipartite graph.

Theorem 2.2. Let $G$ be a connected bipartite graph of order $n$ with bipartition $V(G)=$ $U \cup W, U \cap W=\emptyset,|U|=p$ and $|W|=q$. Then

and (ii)

$$
\begin{aligned}
& E_{1}\left(K_{p, q}\right) \leq E_{1}(G) \leq E_{1}\left(P_{n}\right) \\
& E_{2}\left(K_{p, q}\right) \leq E_{2}(G) \leq E_{2}\left(P_{n}\right) .
\end{aligned}
$$

Moreover, the left hand side (right hand side, respectively) equality holds in (2.3) and (2.4) if and only if $G \cong K_{p, q}\left(G \cong P_{n}\right.$, respectively).

Proof. If $G$ is isomorphic to a complete bipartite graph $K_{p, q}$, then the left hand side equality holds in (2.3) and (2.4). Otherwise, $G \nsubseteq K_{p, q}$. If we add an edge in $G$, then each vertex eccentricity will non-increase. Thus we have $e_{i}(G+e) \leq e_{i}(G)$. Using this property, one can see easily that $E_{1}(G) \geq E_{1}\left(K_{p, q} \backslash\{e\}\right)>E_{1}\left(K_{p, q}\right)$ and $E_{2}(G) \geq E_{2}\left(K_{p, q} \backslash\{e\}\right)>E_{2}\left(K_{p, q}\right)$, where $e$ is any edge in $K_{p, q}$.

Let $T$ be a spanning tree of connected bipartite graph $G$. Then by the above property, $E_{1}(G) \leq E_{1}(T)$ and $E_{2}(G) \leq E_{2}(T)$. Using this result with Theorem 2.1, we get the right hand side inequality in (2.3) and (2.4). Moreover, the right hand side equality holds in (2.3) and (2.4) if and only if $G \cong P_{n}$. This completes the proof.

In [10], Hua et al. proved the following result in Theorem 3.1.

Lemma 2.3. Let $G$ be a connected graph with $e_{i}=n-d_{i}$ for any vertex $v_{i} \in V(G)$. If $G \nsucceq P_{4}$, then $e_{i} \leq 2$ for any vertex $v_{i} \in V(G)$.

We now give some relation between first Zagreb index and the first Zagreb eccentricity index of graphs.

Theorem 2.4. Let $G$ be a connected graph of order $n$ with $m$ edges. Then

$$
E_{1}(G) \leq M_{1}(G)-4 m n+n^{3},
$$

where $M_{1}(G)$ is the first Zagreb index in $G$. Moreover, the equality holds in (2.5) if and only if $G \cong P_{4}$ or $G \cong K_{n}$ or $G$ is isomorphic to a $(n-1, n-2)$-semiregular graph.

Proof. If $G \cong P_{4}$, then the equality holds in (2.5). Otherwise, $G \nsubseteq P_{4}$. Now,

$$
\begin{aligned}
E_{1}(G)=\sum_{v_{i} \in V(G)} e_{i}^{2} & \leq \sum_{v_{i} \in V(G)}\left(n-d_{i}\right)^{2} \text { as } e_{i} \leq n-d_{i} \\
& =M_{1}(G)-4 m n+n^{3} \text { as } M_{1}(G)=\sum_{v_{i} \in V(G)} d_{i}^{2}, \sum_{v_{i} \in V(G)} d_{i}=2 m .
\end{aligned}
$$

First part of the proof is over.

Now suppose that equality holds in (2.5). Then $e_{i}=n-d_{i}$ for all $v_{i} \in V(G)$. By Lemma 2.3, we conclude that $e_{i} \leq 2$ for any vertex $v_{i} \in V(G)$ as $G \nsubseteq P_{4}$. Since $e_{i}=n-d_{i}$ for any vertex $v_{i} \in V(G)$, we must have $d_{i}=n-1$ or $n-2$ for any vertex $v_{i} \in V(G)$, that is, $G \cong K_{n}$ or $G$ is isomorphic to a $(n-1, n-2)$-semiregular graph.

Conversely, one can see easily that (2.5) holds for $P_{4}$ or $K_{n}$ or $(n-1, n-2)$-semiregular graph. 
Remark 2.5. ( $n-1, n-2)$-semiregular graph is obtained by deleting $i$ independent edges from $K_{n}, 1 \leq i \leq\left\lfloor\frac{n}{2}\right\rfloor$.

We now give some relation between first Zagreb index, second Zagreb index and the second Zagreb eccentricity index of graphs.

Theorem 2.6. Let $G$ be a connected graph of order $n$ with $m$ edges. Then

$$
E_{2}(G) \leq M_{2}(G)-n M_{1}(G)+m n^{2},
$$

where $M_{1}(G)$ is the first Zagreb index, $M_{2}(G)$ is the second Zagreb index in $G$. Moreover, the equality holds in (2.6) if and only if $G \cong P_{4}$ or $G \cong K_{n}$ or $G$ is isomorphic to a $(n-1, n-2)$-semiregular graph.

Proof. Now,

$$
\begin{aligned}
E_{2}(G) & =\sum_{v_{i} v_{j} \in E(G)} e_{i} \cdot e_{j} \\
& \leq \sum_{v_{i} v_{j} \in E(G)}\left(n-d_{i}\right)\left(n-d_{j}\right) \text { as } e_{i} \leq n-d_{i} \text { and } e_{j} \leq n-d_{j} \\
& =\sum_{v_{i} v_{j} \in E(G)}\left(n^{2}+d_{i} d_{j}-\left(d_{i}+d_{j}\right) n\right) \\
& =M_{2}(G)-n M_{1}(G)+m n^{2}
\end{aligned}
$$

First part of the proof is over. Moreover, one can see easily that the equality holds in (2.6) if and only if $G \cong P_{4}$ or $G \cong K_{n}$ or $G$ is isomorphic to a $(n-1, n-2)$-semiregular graph, by the proof of Theorem 2.4 .

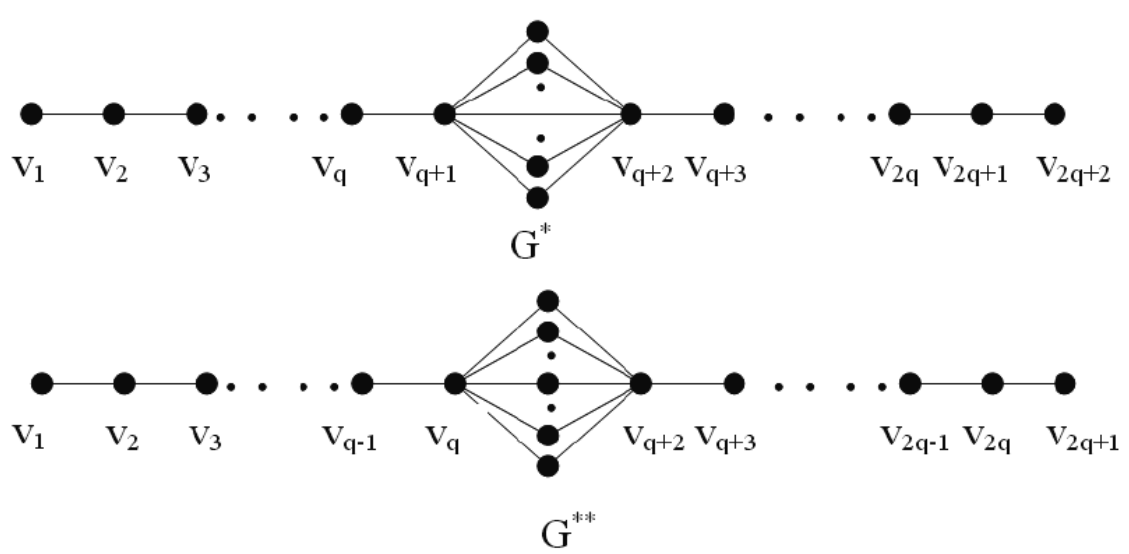

Figure 1: Graphs $G^{*}$ and $G^{* *}$.

Let $K_{2, a-2}^{1}$ be a connected graph of order $a$ obtained from the complete bipartite graph $K_{2, a-2}$ with the vertices of degree $a-2$ are adjacent. Denote by $G^{*}$, is a connected graph 
of order $n$, obtained from $K_{2, n-2 q-2}^{1}$ by attaching two paths $P_{q+1}$ to two of its vertices of degree $n-2 q-1$. Let $\Gamma_{1}$ be the class of graphs $H_{1}=(V, E)$ such that $H_{1}$ is connected graph of diameter $d(d=2 q+1)$ with $V\left(G^{*}\right)=V\left(H_{1}\right)$ and $E\left(G^{*}\right) \subseteq E\left(H_{1}\right)$.

Let $K_{3, a-2}^{2}$ be a connected graph of order $a+1$ obtained from the complete bipartite graph $K_{2, a-2}$ with the vertices of degree $a-2$ are adjacent to a new vertex. Denote by $G^{* *}$, is a connected graph of order $n$, obtained from $K_{3, n-2 q-1}^{2}$ by attaching two paths $P_{q}$ to two of its vertices of degree $n-2 q$. Let $\Gamma_{2}$ be the class of graphs $H_{2}=(V, E)$ such that $\mathrm{H}_{2}$ is connected graph of diameter $d(d=2 q+2)$ with $V\left(G^{*}\right)=V\left(H_{2}\right)$ and $E\left(G^{*}\right) \subseteq E\left(H_{2}\right)$.

We now give another lower bound on $E_{1}(G)$ in terms of $n, d$ and also characterize the extremal graphs.

Theorem 2.7. Let $G$ be a connected graph of order $n$ with diameter $d$. Then

$$
E_{1}(G) \geq \begin{cases}\frac{1}{12}\left(3 n d^{2}+6 n d+3 n+4 d^{3}+3 d^{2}-4 d-3\right) & \text { if } d+1 \text { is even } \\ \frac{d}{12}\left(3 n d+4 d^{2}+9 d+2\right) & \text { if } d+1 \text { is odd }\end{cases}
$$

with equality holding if and only if $G \cong P_{n}$ or $G \in \Gamma_{1}$ or $G \in \Gamma_{2}$.

Proof. Since $G$ has diameter $d, G$ contains a path $P_{d+1}: v_{1} v_{2} \ldots, v_{d+1}$. Moreover, $n \geq$ $d+1$ and $e_{i} \geq\left\lceil\frac{d}{2}\right\rceil, i=1,2, \ldots, n$. If $n=d+1$, then $G \cong P_{n}$ and the equality holds in (2.7). Otherwise, $n>d+1$. By (1.3), we get

$$
\sum_{i=1}^{d+1} e_{i}^{2}= \begin{cases}\frac{d}{12}\left(7 d^{2}+12 d+5\right) & \text { if } d+1 \text { is even } \\ \frac{d}{12}\left(7 d^{2}+12 d+2\right) & \text { if } d+1 \text { is odd }\end{cases}
$$

Since $e_{i} \geq\left\lceil\frac{d}{2}\right\rceil, i=1,2, \ldots, n$, using above result, we get

$$
\begin{aligned}
E_{1}(G) & =\sum_{i=1}^{d+1} e_{i}^{2}+\sum_{i=d+2}^{n} e_{i}^{2} \\
& \geq \begin{cases}\frac{d}{12}\left(7 d^{2}+12 d+5\right)+(n-d-1)\left\lceil\frac{d}{2}\right\rceil^{2} & \text { if } d+1 \text { is even } \\
\frac{d}{12}\left(7 d^{2}+12 d+2\right)+\frac{1}{4}(n-d-1) d^{2} & \text { if } d+1 \text { is odd }\end{cases}
\end{aligned}
$$

from which we get the required result (2.7). First part of the proof is over.

Now suppose that equality holds in (2.7) with $n>d+1$. From equality in (2.9), we get

$$
e_{i}=\left\lceil\frac{d}{2}\right\rceil \text { for } i=d+2, d+3, \ldots, n .
$$

Using above result we conclude that all the vertices $v_{d+2}, v_{d+3}, \ldots, v_{n-1}$ and $v_{n}$ are adjacent to vertices $v_{q}$ and $v_{q+2}$ (when $d=2 q$ ), or $v_{q+1}$ and $v_{q+2}($ when $d=2 q+1$ ). Hence $G \in \Gamma_{1}$ or $G \in \Gamma_{2}$.

Conversely, one can see easily that (2.7) holds for path $P_{n}$ or graph $G$, where $G \in \Gamma_{1}$ or $G \in \Gamma_{2}$. 
We now give another lower bound on $E_{2}(G)$ in terms of $m, d$ and also characterize the extremal graphs.

Theorem 2.8. Let $G$ be a connected graph of order $n$ with diameter $d$. Then

$$
E_{2}(G) \geq \begin{cases}\frac{1}{12}\left(3 m d^{2}+6 m d+4 d^{3}-6 d^{2}-4 d+3 m+6\right) & \text { if } d+1 \text { is even } \\ \frac{d}{12}\left(3 m d+4 d^{2}-4\right) & \text { if } d+1 \text { is odd }\end{cases}
$$

with equality holding if and only if $G \cong P_{n}$ or $G \in \Gamma_{1}$ or $G \in \Gamma_{2}$.

Proof. By (1.3), we get

$$
\sum_{v_{i} v_{j} \in E\left(P_{d+1}\right)} e_{i} e_{j}= \begin{cases}\frac{d+1}{12}\left(7 d^{2}-7 d+6\right) & \text { if } d+1 \text { is even } \\ \frac{d}{12}\left(7 d^{2}-4\right) & \text { if } d+1 \text { is odd }\end{cases}
$$

Since $e_{i} \geq\left\lceil\frac{d}{2}\right\rceil, i=1,2, \ldots, n$, we have

$$
\begin{aligned}
E_{2}(G) & =\sum_{v_{i} v_{j} \in E\left(P_{d+1}\right)} e_{i} e_{j}+\sum_{v_{i} v_{j} \in E\left(G \backslash P_{d+1}\right)} e_{i} e_{j} \\
& \geq \begin{cases}\frac{d+1}{12}\left(7 d^{2}-7 d+6\right)+(m-d)\left\lceil\frac{d}{2}\right\rceil^{2} & \text { if } d+1 \text { is even } \\
\frac{d}{12}\left(7 d^{2}-4\right)+\frac{1}{4}(m-d) d^{2} & \text { if } d+1 \text { is odd, }\end{cases}
\end{aligned}
$$
2.7 .

from which we get the required result (2.10). Rest of the proof is similar as Theorem

Acknowledgement. The authors are grateful to the two anonymous referees for their careful reading of this paper and strict criticisms, constructive corrections and valuable comments on this paper, which have considerably improved the presentation of this paper. The first author's research is supported by Sungkyunkwan University BK21 Project, BK21 Math Modeling HRD Div. Sungkyunkwan University, Suwon, Republic of Korea.

\section{References}

[1] S. J. Cyvin and I. Gutman, Kekulé Structures in Benzenoid Hydrocarbons, Lecture Notes in Chemistry, Vol 46, Springer Verlag, Berlin, 1988.

[2] K. C. Das, Maximizing the sum of the squares of the degrees of a graph, Discrete Math. 285 (2004), 57-66.

[3] K. C. Das and I. Gutman, Some Properties of the Second Zagreb Index, MATCH Commun. Math. Comput. Chem. 52 (2004), 103-112.

[4] K. C. Das, I. Gutman and B. Zhou, New upper bounds on Zagreb indices, J. Math. Chem. 46 (2009), 514-521.

[5] M. Ghorbani and M. A. Hosseinzadeh, A new version of Zagreb indices, Filomat 26 (2012), 93-100.

[6] A. Graovac, I. Gutman and N. Trinajstić, Topological Approach to the Chemistry of Conjugated Molecules, Springer Verlag, Berlin, 1977.

[7] I. Gutman and K. C. Das, The first Zagreb indices 30 years after, MATCH Commun. Math. Comput. Chem. 50 (2004), 83-92. 
[8] I. Gutman and O. E. Polansky, Mathematical Concepts in Organic Chemistry, Springer Verlag, Berlin, 1986.

[9] I. Gutman and N. Trinajstić, Graph theory and molecular orbitals. III. Total $\pi$-electron energy of alternant hydrocarbons, Chem. Phys. Lett. 17 (1972), 535-538.

[10] H. Hua and S. Zhang, Relations between Zagreb coindices and some distance-Based topological indices, MATCH Commun. Math. Comput. Chem. 68 (2012), 199-208.

[11] M. Randić, On characterization of molecular branching, J. Am. Chem. Soc. 97 (1975), 6609 6615.

[12] D. Vukičević and A. Graovac, Note on the comparison of the first and second normalized Zagreb eccentricity indices, Acta Chim. Slov. 57 (2010), 524-528.

[13] R. Todeschini and V. Consonni, Handbook of Molecular Descriptors, Wiley-VCH, Weinheim, 2000.

[14] N. Trinajstić and I. Gutman, Mathematical chemistry, Croat. Chem. Acta 75 (2002), 329-356. 